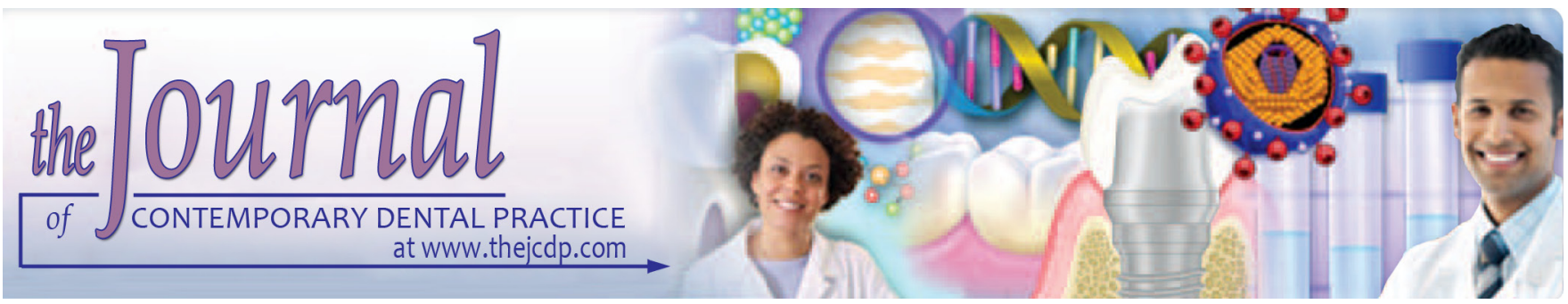

\title{
Effects of Baseplates of Orthodontic Appliances with in situ generated Silver Nanoparticles on Cariogenic Bacteria: A Randomized, Double- blind Cross-over Clinical Trial
}

${ }^{1}$ Roghayeh Ghorbanzadeh, ${ }^{2}$ Babak Pourakbari, ${ }^{3}$ Abbas Bahador

\begin{abstract}
Aim: Polymethyl-methacrylate (PMMA) is commonly used primarily for baseplates of orthodontic appliances (BOA). The activities of cariogenic bacteria in biofilm on these surfaces may contribute to dental caries, gingival inflammation and periodontal disease. The PMMA incorporated with nanoparticles of silver (NanoAg-I-PMMA) and NanoAg in situ in PMMA (NanoAg-IS-PMMA) have been shown to control the growth of cariogenic bacteria, but clinical trial of anti-cariogenic application of these novel materials in orthodontics has not been evaluated. The main aim of the study is to compare the clinical effectiveness of using NanoAg-IS-PMMA and NanoAg-I-PMMA for construction of new BOA in inhibiting the planktonic growth and biofilm formation of the cariogenic bacteria.
\end{abstract}

Materials and methods: Twenty four patients with a median age of 12.6 years (7-15) harboring Streptococcus mutans, Streptococcus sobrinus and Lactobacillus acidophilus as well as Lactobacillus casei participated in the randomized, doubleblind, cross-over study. The experimental BOA, NanoAg-ISBOA and NanoAg-I-BOA, contained $0.5 \%$ w/w NanoAg while the control BOA was standard PMMA. Antibacterial effect of NanoAg-IS-BOA and NanoAg-I-BOA was assessed against test cariogenic bacteria by planktonic and biofilm bacterial cells growth inhibition.

Results: The average levels of test cariogenic bacteria in saliva decreased about 2 to 70 fold (30.9-98.4\%) compared to

\footnotetext{
${ }^{1}$ Private Practices, Tehran, Iran

${ }^{2}$ Pediatrics Infectious Disease Research Center, School of Medicine, Tehran University of Medical Sciences, Tehran, Iran

${ }^{3}$ Department of Medical Microbiology, Faculty of Medicine Tehran University of Medical Sciences, Tehran, Iran

Corresponding Author: Abbas Bahador, Associate Professor Department of Medical Microbiology, Medical Faculty, Tehran University of Medical Sciences, Tehran, Iran, Phone: 009821-77326397, Fax: 0098-21-88955810, e-mail: abahador@ sina.tums.ac.ir
}

baseline depending on the microorganism type and test BOA. Biofilm inhibition analysis demonstrated that NanoAg-I-BOA and NanoAg-IS-BOA inhibited the biofilm of all test bacteria by 20.1 to $79.9 \%$ compared to BOA. NanoAg-IS-BOA had a strong anti-biofilm effect against $S$. mutans, $S$. sobrinus and L. casei. However, NanoAg-I-BOA showed only slight antibiofilm effects on test bacteria. Most notably, at all period of the clinical trial, NanoAg-IS-BOA showed a higher antibacterial activity than NanoAg-I-BOA.

Conclusion: Based on the novel data that presented here, the NanoAg-IS-BOA had strong antimicrobial activity in the planktonic phase and subsequent biofilm formation of the cariogenic bacteria.

Clinical significance: Wearing of NanoAg-IS-BOA has the potential to minimize dental plaque formation and caries during orthodontic treatment.

Keywords: Cariogenic Bacteria, Nano Silver, Orthodontics, Polymethyl-methacrylate.

How to cite this article: Ghorbanzadeh R, Pourakbari B, Bahador A. Effects of Baseplates of Orthodontic Appliances with in situ generated Silver Nanoparticles on Cariogenic Bacteria: A Randomized, Double-blind Cross-over Clinical Trial. J Contemp Dent Pract 2015;16(4):291-298.

\section{Source of support: Nil}

Conflict of interest: None

\section{INTRODUCTION}

Polymethyl-methacrylates (PMMAs) remain the most popular material for use in orthodontics as baseplates of orthodontic appliances (BOA) especially in developing countries because of their low cost and ease of use. ${ }^{1}$ In addition, PMMAs are used for individual impression trays and early orthodontic appliances for patients with cleft lip and palate and as splints for orthognathic surgery. Currently, PMMA resins are commonly used in routine 
orthodontic treatments for construction of removable and auxiliary fixed appliances as well as Hawley retainers. ${ }^{2}$

Unfortunately, regarding the long-term existence of BOA in mouth and their surfaces porosities may have a negative effect on oral microbiota, promote the biofilm formation and may contribute to dental caries, gingival inflammation and periodontal disease. ${ }^{3-5}$ It is challenging which results from higher number of plaque-retentive sites and impaired mechanical plaque removal that most often seen with BOA. ${ }^{6}$ The BOA also may affect the composition, metabolic activity, pathogenicity of the biofilm. ${ }^{7}$

Mechanical cleaning of the BOA is helpful in reducing biofilm and accumulation of microbial plaque, particularly with the adjunctive use of antimicrobial solutions. ${ }^{8,9}$ However, such measures rely generally on conformity of patients, and may not be optimal in pediatric, geriatric, and handicapped individuals. Therefore, an additive that robustly enhances the inhibitory effects of BOA, while maintaining its biocompatibility, is highly desirable. ${ }^{10}$

Silver in nanoparticulate form (NanoAg) is an effective microbicidal agent due to their high surface area-tovolume ratios which attach to the outer membrane and affect permeability as well as induce structural changes in the cell-ultimately leading to cell death. In addition, NanoAg does not cause resistant microbial species to develop. ${ }^{11-13}$ The literature reports various studies related to NanoAg inhibit the bacterial growth of oral bacteria at very low concentrations without side effects. ${ }^{14,15}$

The PMMA incorporated with NanoAg (referred to as NanoAg-I-PMMA) has been reported to reduce adherence and inhibited growth of microorganisms. ${ }^{16,17}$ The PMMA incorporated with NanoAg are also reported to be noncytotoxic and nongenotoxic. ${ }^{17}$ However, the incorporation of NanoAg has been problematic often due to an inability to disperse homogenously NanoAg, which is hurtful to the device's mechanical properties, and the need for harsh chemicals and complex processes required for synthesis, which is unsuitable for dental and medical applications. ${ }^{18-21}$ Recently, Fan et $\mathrm{al}^{22}$ have developed a broad-spectrum, antimicrobial PMMA acryl with welldispersed NanoAg and without the use of harsh chemicals or multistep processes by synthesizing NanoAg in situ in PMMA (referred to as NanoAg-IS-PMMA). In an in vitro pilot study Fan $^{22}$ showed that NanoAg-IS-PMMA inhibited planktonic growth of Streptococcus mutans, the primary causative agent in the formation of dental cavities in human. The activities of cariogenic microorganisms such as S. mutans, Streptococcus sobrinus, Lactobacillus acidophilus and Lactobacillus casei in biofilm on BOA may contribute to dental caries, gingival inflammation and periodontal disease. ${ }^{23-26}$ The coexistence of S. mutans and
S. sobrinus in dental biofilm is associated with higher caries experience than if only $S$. mutans is detected. ${ }^{27}$

To our knowledge no randomized clinical trial has examined with NanoAg-IS-BOA and NanoAg-I-BOA to determine its clinical efficacy to inhibited planktonic growth and biofilm formation of S. mutans and S. sobrinus, $L$. acidophilus as well as $L$. casei as cariogenic bacteria. Therefore, the purpose of this study is to compare the clinical effectiveness of NanoAg-IS-BOA and NanoAgI-BOA to inhibition of planktonic growth and biofilm formation of the cariogenic bacteria.

\section{MATERIALS AND METHODS}

\section{Patients}

Between December 2010 and June 2012, 24 patients of both sexes aged 7 to 15 years who had been referred to the private orthodontic clinic (Tehran, Iran) for treatment with removable orthodontic appliances were enrolled in this study. The inclusion and exclusion criteria were as reported in Table 1. The included patients were chosen on the basis of a pre-screening for the presence of $S$. mutans, S. sobrinus, L. acidophilus as well as L. casei. In this research project, written informed consent was obtained from the parents or legal representatives.

\section{Experimental and Control Baseplates of Orthodontic Appliances}

Baseplates of orthodontic appliances without wire clasps were constructed on cast models from each patient using autopolymerizing PMMAs resin (Selecta Plus; Dentsply) following the monomer-to-polymer ratio advised by the manufacturer and standard acrylization, trimming and finishing-polishing techniques.

The NanoAg in situ in BOA (referred to as NanoAgIS-BOA) was prepared using the Fan method..$^{22}$ Briefly, $0.5 \% \mathrm{w} / \mathrm{w}$ of total monomer (Sigma-Aldrich, Germany) of silver benzoate $(\mathrm{AgBz})$ were dissolved in dimethylaminoethyl methacrylate (DMAEMA; 2\% w/w of total monomer; Sigma-Aldrich, Germany). This was then blended with liquid orthodontic monomer (Dentsply, UK), and subsequently mixing with PMMA powder (Selecta Plus; Dentsply, UK) as described in the manufacturer's guidelines.

The procedure for preparation of BOA incorporated with NanoAg (referred to as NanoAg-I-BOA) has been described previously. ${ }^{28}$ Briefly, for preparation of NanoAg, aqueous silver nitrate $(100 \mu \mathrm{l}, 0.1 \mathrm{M})$ was added to isopropyl alcohol $(2 \mathrm{ml})$. The suspension was centrifuged after 30 minutes vigorous stirring at room temperature and washed with ethanol. Prepared NanoAg at $0.5 \%$ w/w of total monomer (Sigma Aldrich, 
Table 1: Inclusion and exclusion criteria for participation in this study

\begin{tabular}{l}
\hline Inclusion criteria \\
\hline - Subject giving his written informed consent \\
- Subject considered as normal after clinical examination \\
- Subject willing to comply with the study procedures \\
Exclusion criteria \\
- Systemic disease \\
- Infectious or inflammatory diseases in the last month \\
- Taking medicine: antibiotics or fluoride in the last month \\
- Habitual consumers of xylitol products and sorbitol-containing \\
products \\
- Mouth rinses \\
- Abnormal salivary flow rates of paraffin-stimulated saliva \\
(<1 ml/min) \\
- Pregnancy \\
- Contraceptive pill \\
- Missed and filled tooth (MDFT) one filled and/or decayed \\
$\quad$ (in dentine) \\
- Abnormal or particular dietary habits \\
- Consumption of food complements: antioxidant,...
\end{tabular}

Germany) were added to the acryl liquid and sonicated for 15 minutes, and subsequently mixing with PMMA powder (Selecta Plus; Dentsply) as described in the manufacturer's instruction. The control BOA constructed with the standard PMMA (Selecta Plus, Dentsply, UK).

\section{Study Design}

The investigation had a cross-over randomized doubleblind prospective design with two arms (Flow Chart 1). ${ }^{29}$ The study and intervention involved were completely explained to all participants. Following screening, patients were randomly allocated into one of two groups. Patients entered a 4 weeks washout period followed by a 4 weeks treatment period (Group AB/part 1). At the end of this period, there was a 4 weeks washout period. Patients then entered a second 4 weeks treatment period when they received the alternative treatment (Group BA/part 2). Group AB consisted of NanoAg-IS-BOA for 4 weeks followed by 4 weeks wash out then matched NanoAg-IBOA as the same as NanoAg-IS-BOA. Group BA consisted of NanoAg-I-BOA for 4 weeks, a 4 weeks period washout, and then NanoAg-IS-BOA for a 4 weeks period. The BOA constructed with standard PMMA were used during the washout periods.

The patients were requested to wear the BOA full-time, including during sleep. During this time the patients were educated not to use mouthwashes, antibiotics or xylitol products, but to continue their usual tooth brushing and consume their normal diet. The patients were instructed not to use tooth brushing and other oral hygiene procedures for 24 hours before sample collection and not to eat or drink for 1 hour before the sampling. At the appointments, the patients were also interviewed about confounding factors like acute infectious diseases and use of antibiotics. The patients and researchers in this study were blinded throughout the study. Also, the microbiological analyses in Tehran University of Medical Sciences (TUMS) were carried out blinded.

\section{Planktonic Bacterial Cells Growth Inhibition}

Planktonic microorganism's growth inhibition was determined using the Bahador method ${ }^{29}$ with some modifications. The pre-experimental and postexperimental saliva were collected into sterile Flat-top cap for BD Falcon ${ }^{\mathrm{TM}} 50 \mathrm{ml}$ conical centrifuge tubes (Becton, Dickinson; Tokyo, Japan). The saliva samples were homogenized by ultra-sonication, $20 \mu \mathrm{l}$ aliquots of 10 -fold diluted samples were plated on the selective media. Mitis S. mutans valinomycin (MS-MUTV) agar, Mitis S. sobrinus (MS-SOB) agar, L. casei (LC) agar and De Man Rogosa Sharpe agar containing maltose (MRSM) was used for detection of S. mutans, S. sobrinus and L. casei as well as L. acidophilus, respectively. After 72 hours of incubation at $37^{\circ} \mathrm{C}$ in an anaerobic atmosphere $\left(5 \% \mathrm{CO}_{2}, 10 \% \mathrm{H}_{2}\right.$, and $85 \% \mathrm{~N}_{2}$ ), colony-forming units ( $\mathrm{CFU}$ ) were enumerated for the estimation of test microorganisms levels on their selective media. For confirmation of the selectivity of media, colonies were identified biochemically using a rapid ID 32 STREP system (bioMérieux, France).

\section{Biofilm Bacterial Cells Growth Inhibition}

The biofilm inhibition potential of NanoAg-IS-BOA and NanoAg-I-BOA was evaluated using the membrane filter (MF) technique with some modifications. ${ }^{30}$ Briefly, the BOA gently washed with sterile PBS three times to remove the planktonic cells and loosely adherent bacteria. Biofilm forming bacteria on the $\mathrm{BOA}$ were dislodged by ultrasonication ( $5 \mathrm{~min}$ ) in a $150 \mathrm{~W}$ ultrasonic bath (Branson Ultrasonics Co, Shanghai, China) operating at a frequency of $50 \mathrm{~Hz}$. Ultrasonication was followed by rapid vortex mixing (Scientific Industries, Bohemia, NY, USA) at maximum power for 1 minute to remove bacteria that had adhered to the material. This method is known to be effective for removing biomaterial-adherent bacteria. ${ }^{17}$ Finally, a microbial cell viability assay based on the MF technique was performed in order to determine the number of viable cells adhered to BOA This method consists of filtering a suspension sample on a sterile filter with a $0.45 \mu \mathrm{m}$ pore size which retains bacteria, incubating this filter on a selective medium and enumerating typical colonies on the filter. 
Table 2: The effect of NanoAg-IS-BOA vs NanoAg-I-BOA on planktonic growth of the cariogenic bacteria. The counts (log 10 CFU/ml) of each microorganism per saliva sample are expressed as logarithmic values of mean $\pm \mathrm{SD}$, minimum, maximum and variance

\begin{tabular}{|c|c|c|c|c|c|c|}
\hline \multirow[b]{2}{*}{ Microorganisms } & \multicolumn{3}{|c|}{ NanoAg-IS-BOA } & \multicolumn{3}{|c|}{ NanoAg-I-BOA } \\
\hline & Baseline & After wear & Mean percent reduction & Baseline & After wear & Mean percent reduction \\
\hline S. mutans & & & $\dagger$ & & & $\dagger$ \\
\hline Mean & 3.05 & 1.23 & 98.4 & 2.99 & 2.13 & 86.1 \\
\hline SD & 1.38 & 0.78 & $(p=0.004) \dagger$ & 1.16 & 0.90 & $(p=0.017) \dagger$ \\
\hline Minimum & 0.39 & 0.00 & & 1.10 & 0.8 & \\
\hline Maximum & 5.45 & 3.20 & & 5.14 & 4.08 & \\
\hline Variance & 1.91 & 0.61 & & 1.35 & 0.81 & \\
\hline S. sobrinus & & & $\dagger$ & & & $\dagger$ \\
\hline Mean & 2.60 & 1.54 & 91.6 & 2.35 & 1.55 & 84.3 \\
\hline SD & 1.18 & 0.71 & $(p=0.023) \dagger$ & 1.11 & 0.74 & $(p=0.025) \dagger$ \\
\hline Minimum & 0.53 & 0.57 & & 0.40 & 0.20 & \\
\hline Maximum & 4.61 & 3.04 & & 4.20 & 2.84 & \\
\hline Variance & 1.42 & 0.51 & & 1.25 & 0.55 & \\
\hline L. acidophilus & & & $\dagger$ & & & \\
\hline Mean & 4.14 & 3.59 & 70.2 & 3.88 & 3.69 & 34.7 \\
\hline SD & 1.44 & 1.04 & $(p=0.014) \dagger$ & 1.22 & 0.99 & $(p=0.832)$ \\
\hline Minimum & 1.20 & 0.70 & & 0.81 & 0.41 & \\
\hline Maximum & 6.11 & 4.10 & & 5.85 & 4.92 & \\
\hline Variance & 2.08 & 1.09 & & 1.49 & 0.99 & \\
\hline L. casei & & & $\dagger$ & & & \\
\hline Mean & 2.24 & 1.71 & 71.1 & 2.51 & 2.35 & 30.9 \\
\hline SD & 1.31 & 0.91 & $(p=0.005) \dagger$ & 0.88 & 1.09 & $(p=0.410)$ \\
\hline Minimum & 0.31 & 0.00 & & 0.64 & 0.42 & \\
\hline Maximum & 4.34 & 3.34 & & 4.11 & 4.53 & \\
\hline Variance & 1.72 & 0.82 & & 0.79 & 1.19 & \\
\hline
\end{tabular}

† denotes a statistically significant difference, SD: Standard deviation

\section{Statistical Analysis}

Since bacterial (CFU)/ml did not exhibit a normal distribution, the data were transformed to logarithms to confer homogeneity among the groups and then submitted to variance analysis with repeated measures. The data concerning S. mutans, S. sobrinus, L. acidophilus as well as L. casei salivary levels at the four sampling phases were analyzed for a normal distribution. Differences between groups were assessed using the ANOVA test. The level of statistical significance was set at $\mathrm{p}<0.05$. The statistical software package used was SPSS 14.0 (SPSS Inc, Chicago, Ill, USA). For statistical analyses, where no bacterium detected, the levels of detection limit were $50 \mathrm{CFU} / \mathrm{ml}$ for each bacterial species. ${ }^{29}$

\section{RESULTS}

Twenty-four (15 females and 9 males) of 58 Pre-included patients, with a median age of 12.6 years (7-15) completed the study. Seven persons excluded on antimicrobial therapy and dietetic criteria (Flow Chart 2).

\section{Planktonic Bacterial Cells Growth Inhibition}

After the experimental period the average levels of test cariogenic bacteria in saliva decreased about 2 to 70 fold depending on the microorganism type and $\mathrm{BOA}$ (Table 2). Most notably, NanoAg-IS-BOA and NanoAg-I-BOA showed a higher inhibitory activity than untreated BOA. As shown in Table 2, exposure to NanoAg-IS-BOA had a marked antibacterial effect on tested bacteria, with a reduction in planktonic bacterial cells viability by 70.2 to $98.4 \%$ compared to baseline. In planktonic bacterial cells growth of test bacteria except L. acidophilus, differences between the antibacterial effects of NanoAg-I-BOA vs those with untreated BOA were statistically significant $(\mathrm{p}<0.05)$ with a reduction in cells viability by 30.9 to $86.1 \%$, compared to baseline.

The mean percentage reduction in logarithmic value for L. acidophilus between the test groups and the BOA groups was lower than the percentages for the others (34.7-70.2\%). In S. mutans salivary levels, the mean percentage of logarithmic value in the NanoAg-IS-BOA and NanoAg-I-BOA groups dropped to 1.6 and $13.9 \%$, respectively, after 4 weeks, and this difference was statistically significant $(\mathrm{p}=0.001$ and $\mathrm{p}=0.017)$ in comparison to those in the control/BOA groups. On the other hand, after a 4 weeks exposure NanoAg-IS-BOA inhibited the growth of all test planktonic bacteria by 2 to $40.2 \%$ compared to NanoAg-I-BOA. The planktonic bacterial cells growth inhibition by NanoAg-IS-BOA was significant compared 
Flow Chart 1: Algorithm of the study design. Yr: year; W: week; NanoAg-IS-BOA: NanoAg in situ in baseplates of orthodontic appliances (BOA); NanoAg-I- BOA: BOA incorporated with NanoAg

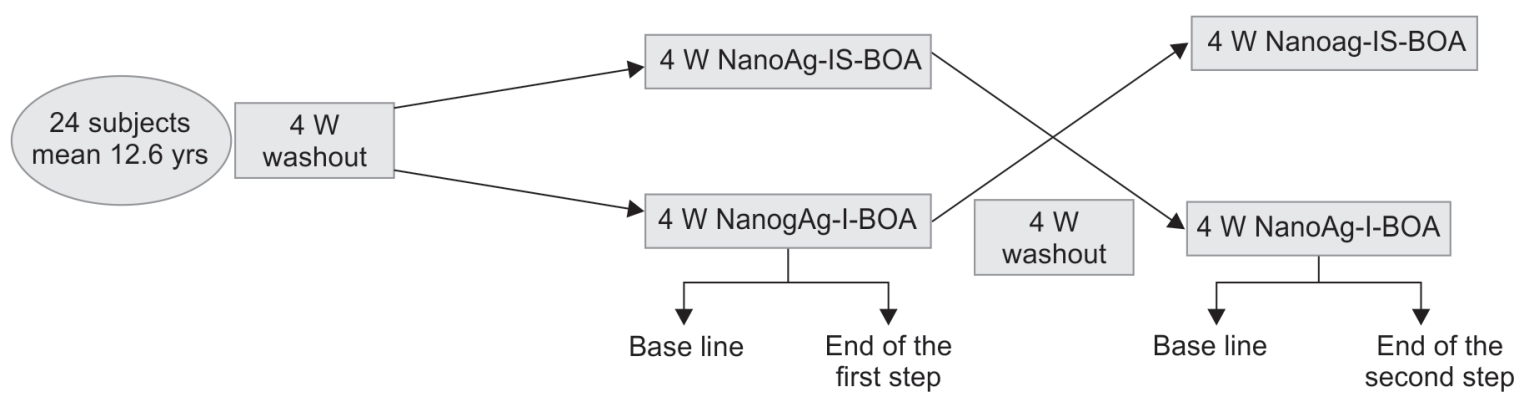

Flow Chart 2: The patients in the study

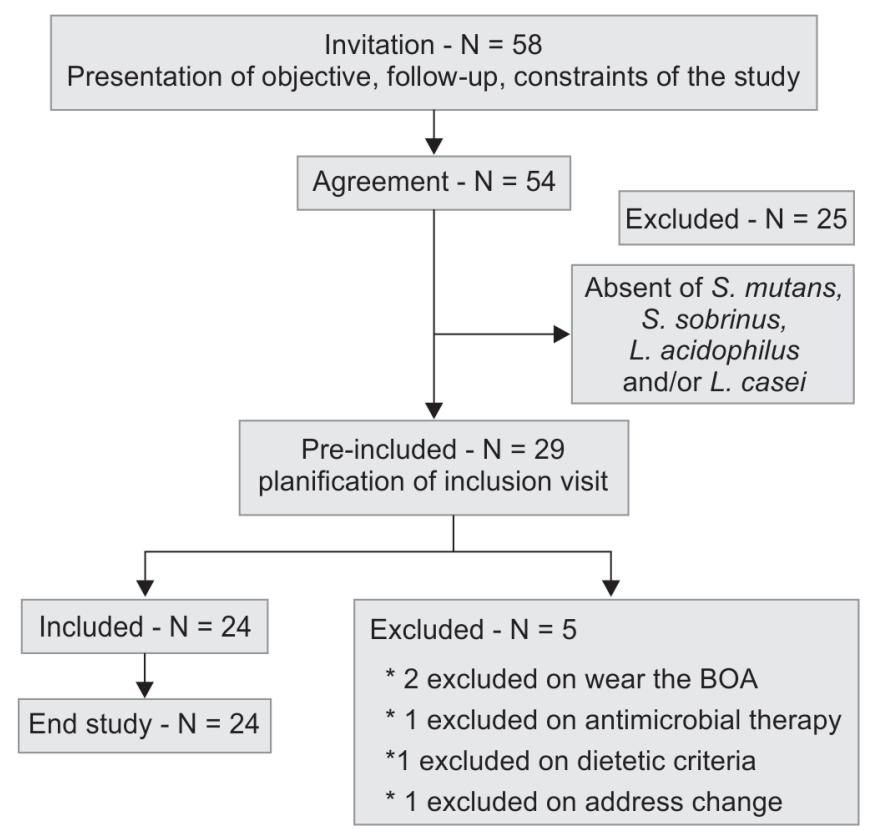

to NanoAg-I-BOA against L. casei ( $\mathrm{p}=0.038)$, as shown in Table 2.

\section{Biofilm Inhibition Potential of Acrylic Disks}

Table 3 summarizes the number of CFU/BOA of tested bacteria after 4 weeks biofilm growth on NanoAg-ISBOA and NanoAg-I-BOA, as a function of NanoAg as expressed relative to the maximal number of $\mathrm{CFU} / \mathrm{BOA}$ recovered from the corresponding control BOA without Ag-NPs. The number of CFU/BOA on the acryl disks without Ag-NPs had a maximum of about $4 \mathrm{CFU} / \mathrm{BOA}$ to $1.05 \times 10^{2} \mathrm{CFU} / \mathrm{BOA}$, which was dependent of the types of tested bacteria (Table 3 ). This maximum was used as the $100 \%$ level for each tested bacteria as well as mixed cultures of the four species in this study.

The results obtained show that NanoAg-IS-BOA and NanoAg-I-BOA caused a significant reduction in the formation of colonies/biofilms of test microorganism on the BOAs surface over the test period ( $p<0.05)$. Biofilm inhibition analysis demonstrated that NanoAg-I-BOA and NanoAg-IS-BOA inhibited the biofilm of all test bacteria by 20.1 to $79.9 \%$ compared to BOA. Although
L. acidophilus colonies/biofilms were not found on the three baseplates of the NanoAg-IS-BOA groups (12.5\%), as shown in Table 3, biofilm of L. acidophilus showed the lowest susceptibility to NanoAg-IS-BOA, which reduced bacterial viability by $70.2 \%(p=0.0641)$. Biofilm inhibition analysis demonstrated that NanoAg-IS-BOA inhibited statistically significant the biofilm growth of S. mutans, S. sobrinus, and L. casei by 26.8 to $54 \%$ compared to NanoAg-I-BOA (Table 3). Our study demonstrated a significantly lower number of viable cells of test bacteria except L acidophilus on the NanoAg-IS-BOA and NanoAgI-BOA compared with the control BOAs by the end of the experiment.

\section{DISCUSSION}

Colonization and plaque formation of cariogenic bacteria on BOA may result in biofilm formation and may contribute to dental caries, gingival inflammation and periodontal disease. ${ }^{23-25,31}$ The use of PMMA containing or coating with antimicrobial agents is advocated

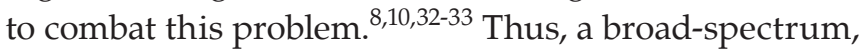
antimicrobial resin without the risk of developing resistant species of bacteria is needed as appliances to prevent post-treatment adverse effects. In recent years, much focus has been given to the potential role of nanoparticles-containing or coating orthodontic appliances in inhibiting the growth of the cariogenic bacteria. The application of nanoparticles for the control of cariogenic biofilm on BOA has not been widely investigated. Herein we performed randomized study to compare the efficiency of anti-cariogenic activity of NanoAg-IS-BOA and NanoAg-I-BOA in terms of orthodontic treatment time by using inhibition of cariogenic planktonic and biofilm bacteria.

This study is part of a series to explore the selective NanoAg activity that can be used as anti-cariogenic agent. Kassaee et $\mathrm{al}^{28}$ showed a strong antibacterial activity of PMMA incorporated with NanoAg (NanoAg-I-PMMA) as a new dental acrylic resin against E. coli. In a pilot in vitro study, although NanoAg in situ in PMMA (NanoAg-IS-PMMA) has been reported to 
Table 3: The effect of NanoAg-IS-BOA vs NanoAg-I-BOA on biofilm formation of the cariogenic bacteria. The counts (log 10 CFU/ml) of each microorganism per BOA sample are expressed as logarithmic values of mean $\pm \mathrm{SD}$, minimum, maximum and variance

\begin{tabular}{|c|c|c|c|c|c|c|}
\hline \multirow[b]{2}{*}{ Microorganisms } & \multicolumn{3}{|c|}{ NanoAg-IS-BOA } & \multicolumn{3}{|c|}{ NanoAg-I-BOA } \\
\hline & Baseline & After wear & Mean percent reduction & Baseline & After wear & Mean percent reduction \\
\hline \multicolumn{7}{|l|}{ S. mutans } \\
\hline Mean & 1.95 & 1.37 & \multirow{5}{*}{$\begin{array}{l}74.1 \\
(p=0.010) \dagger\end{array}$} & 1.55 & 1.46 & \multirow{5}{*}{$\begin{array}{l}20.1 \\
(p=0.276)\end{array}$} \\
\hline $\mathrm{SD}$ & 0.75 & 0.92 & & 0.89 & 0.72 & \\
\hline Minimum & 0.21 & 0.00 & & 0.22 & 0.16 & \\
\hline Maximum & 3.41 & 3.08 & & 3.20 & 3.01 & \\
\hline Variance & 0.56 & 0.86 & & 0.85 & 0.52 & \\
\hline \multicolumn{7}{|l|}{ S. sobrinus } \\
\hline Mean & 2.02 & 1.32 & \multirow{5}{*}{$\begin{array}{l}79.9 \\
(p=0.009) \dagger\end{array}$} & 1.86 & 1.73 & \multirow{5}{*}{$\begin{array}{l}25.4 \\
(p=0.92)\end{array}$} \\
\hline SD & 1.07 & 0.84 & & 0.86 & 0.74 & \\
\hline Minimum & 0.53 & 0.00 & & 0.40 & 0.20 & \\
\hline Maximum & 3.72 & 2.91 & & 3.62 & 2.84 & \\
\hline Variance & 1.15 & 0.70 & & 0.76 & 0.55 & \\
\hline \multicolumn{7}{|l|}{ L. acidophilus } \\
\hline Mean & 1.32 & 1.11 & \multirow{5}{*}{$\begin{array}{l}40.3 \\
(p=0.94)\end{array}$} & 1.02 & 0.95 & \multirow{5}{*}{$\begin{array}{l}27.2 \\
(p=0.18)\end{array}$} \\
\hline SD & 0.76 & 0.65 & & 0.47 & 0.37 & \\
\hline Minimum & 0.20 & 0.00 & & 0.31 & 0.15 & \\
\hline Maximum & 2.72 & 2.60 & & 1.9 & 1.7 & \\
\hline Variance & 0.74 & 0.43 & & 0.22 & 0.29 & \\
\hline \multicolumn{7}{|l|}{ L. casei } \\
\hline Mean & 0.61 & 0.19 & \multirow{5}{*}{$\begin{array}{l}61.5 \\
(p=0.031) \dagger\end{array}$} & 0.67 & 0.47 & \multirow{5}{*}{$\begin{array}{l}34.7 \\
(p=0.086)\end{array}$} \\
\hline SD & 0.22 & 0.33 & & 0.41 & 0.32 & \\
\hline Minimum & 0.21 & 0.00 & & 0.15 & 0.10 & \\
\hline Maximum & 1.10 & 0.90 & & 1.8 & 1.10 & \\
\hline Variance & 0.04 & 0.10 & & 0.17 & 0.19 & \\
\hline
\end{tabular}

† denotes a statistically significant difference, SD: Standard deviation

significantly reduce the $S$. mutans levels; ${ }^{22}$ its effect against cariogenic bacteria in a clinical trial has not been explored. Another study in this series showed the effectiveness of NanoAg-IS-PMMA on the A. bumannii, $P$. aeruginosa, $P$. mirabilis and $S$. aureus. ${ }^{34}$ In the present study, we tested the efficiency of NanoAg in situ in baseplates of orthodontic appliances (NanoAg-IS-BOA) and BOA incorporated with NanoAg (NanoAg-I-BOA) in reducing planktonic and biofilm load of $S$. mutans, S. sobrinus, L. acidophilus as well as L. casei, as cariogenic agents in randomized controlled clinical trials.

Our results showed that 4 weeks wearing of NanoAgIS-BOA and NanoAg-I-BOA reduced significantly the planktonic levels of test bacteria in saliva compared to baseline levels. However, NanoAg-IS-BOA had a higher antibacterial activity than NanoAg-I-BOA against the cariogenic bacteria. For instance, unlike NanoAg-ISBOA, NanoAg-I-BOA did not significantly affect the L. acidophilus level in saliva and biofilm formation. It is possible that the activities were influenced by the NanoAg dispersing and homogeneous incorporation into the resin. Many studies have demonstrated the addition of NanoAg in to PMMA for production of NanoAg-I-PMMA has been problematic often due to an inability to disperse homogenously NanoAg, which is detrimental to the device's mechanical properties, and the need for harsh chemicals and complex multistep processes required for synthesis which is unsuitable for medical and dental applications. ${ }^{18-21}$ Recently, Fan et $\mathrm{al}^{22}$ used the novel approach to generate NanoAg in situ in PMMA (NanoAg-IS-PMMA). The NanoAg-IS-PMMA were eliminated the problem of non-uniform NanoAg dispersion as well as the need for harsh chemicals. This NanoAg-IS-PMMA showed excellent antimicrobial properties against planktonic bacterial cells growth of A. bumannii, P. aeruginosa, $P$. mirabilis, $S$. aureus and $S$. mutans. ${ }^{34}$ Our data demonstrated the NanoAg-IS-BOA exhibited a high antimicrobial efficacy, even if the content of silver nanoparticles in NanoAg-IS-BOA is as low as $0.5 \% \mathrm{w} / \mathrm{w}$ all test bacteria in saliva are inhibited over 53.2 to $98.4 \%$ within 4 weeks. This low amount of NanoAg does not have any adverse effect on the mechanical properties of the polymer. ${ }^{16}$

Aside from NanoAg-IS-BOA, studies involving NanoAg-I-BOA suggests that it has little effect on actively reducing cariogenic bacteria levels and did not effect on in vivo biofilm formation of test bacteria. However, the effect of NanoAg-IS-BOA was lower for test cariogenic bacteria growing in biofilm compared with cells in saliva or planktonic counterparts (at least 2-fold). In the present 
study, complete elimination of biofilm did not occur, since biofilm structures represent an anchor structure and protection for microorganisms. Our biofilm inhibition data seems consistent with several studies that have reported of increase of biofilm resistance to antimicrobial agents compared with their planktonic counterparts. ${ }^{35,36}$ This makes biofilms of cariogenic bacteria particularly disturbing in tooth health, while their presence often creates dental caries. ${ }^{23}$ Mechanisms proposed to increase of biofilm resistance to antimicrobial agents compared with their planktonic counterparts can be divided into three categories: transport limitation, modulation of the environment and new genes expression. ${ }^{37}$ The NanoAg initially killing the microorganisms and the recovery in cell number is then due to the dead bacteria forming a layer through which the Ag ions must diffuse. However, the exopolymers matrix excludes and/or deters the access of agents to microorganisms within a biofilm. ${ }^{38}$ Some cells may differentiate into a new phenotype once attached to a surface, which gives them increased resistance. These resistant bacteria survive, multiply, and result in an increase in the viable cell count of the bacterial biofilm. The limitation of nutrients within the biofilm leads to a decreased growth rate of bacteria and results in a gradient of antimicrobial agents sensitivity from the upper layers to the innermost layers of biofilm, where the metabolism and targets of microorganisms may be altered. ${ }^{39}$ Change in gene expression in biofilm forming bacteria could then influence the susceptibility of bacteria to biocide. ${ }^{40}$

In this NanoAg-IS-BOA time course study, it exhibited a wide spectrum inhibition of planktonic and biofilm bacterial cells growth of $S$. mutans, S. sobrinus and L. casei, the cariogenic bacteria. An advantage of a wide spectrum bactericidal action may be that it permits trial to proceed without bacterial interference and reduces the likelihood for resistance to develop. The varied and broad spectrum activity of NanoAg in vitro has been reported before. ${ }^{41}$ Based on results of the present study, the strong antimicrobial activity of NanoAg-IS-BOA in comparison to NanoAg-I-BOA and PMMA, regarding other studies which have shown considering non-detrimental effects of NanoAg-IS-BOA on mechanical properties of acrylic, ${ }^{22,34}$ it seems clinically advantageous to use NanoAg-IS-BOA and benefit their antimicrobial properties. However, further studies are needed to evaluate the proper concentrations for final products. We acknowledge that further evaluation of the anticariogenic activity of NanoAg-ISBOA against additional common cariogenic agents, such as $A$. naeslundii will enhance our study, since $A$. naeslundii has the potential to invade dentinal tubules and is associated with root caries. ${ }^{42}$

\section{CONCLUSION}

In the present randomized, double-blind cross-over clinical trial, we have shown that the NanoAg-IS-BOA has strong antimicrobial activity in the planktonic phase and subsequent biofilm formation of cariogenic bacteria than NanoAg-I-BOA. It shows that wearing of NanoAgIS-BOA has the potential to minimize plaque formation of cariogenic bacteria and dental carise.

\section{ACKNOWLEDGMENTS}

The authors are grateful to Dr Reza Raufian for his technical assistance. This study was in part supported by Department of Microbiology and Medical School Research Committee at Tehran University of Medical Sciences (TUMS).

\section{REFERENCES}

1. Siqueira-Gonçalves $\mathrm{T}$, Schmitt MV, Thomas M, Lopes de Souza MA, Macedo de Menezes L. Cytotoxicity of two autopolymerized acrylic resins used in orthodontics. Angle Orthodont 2008;78(5):926-930.

2. Öztürk F, Malkoc S, Ersöz M, Hakki SS, Bozkurt BS. Realtime cell analysis of the cytotoxicity of the components of orthodontic acrylic materials on gingival fibroblasts. Am J Orthod Dentofacial Orthop 2011;140(5):e243-e249.

3. Diamanti-Kipioti A, Gusberti FA, Lang NP. Clinical and microbiological effects of fixed orthodontic appliances. J Clin Periodontol 1987;14(6):326-333.

4. Topaloglu-Ak A, Ertugrul F, Eden E, Ates M, Bulut H. Effect of orthodontic appliances on oral microbiota-six months follow-up. J Clin Pediatr Dent 2011;35(4):433-436.

5. Naranjo AA, Triviño ML, Jaramillo A, Betancourth M, Botero JE. Changes in the subgingival microbiota and periodontal parameters before and 3 months after bracket placement. Am J Orthod Dentofacial Orthop 2006;130(3):275. e17-e22.

6. Eliades T, Eliades G, Brantley WA. Microbial attachment on orthodontic appliances: ability and early pellicle formation on bracket materials. Am J Orthod Dentofacial Orthop 1995;108(4):351-360.

7. Atack NE, Sandy JR, Addy M. Periodontal and microbiological changes associated with the placement of orthodontic appliances. A Review. J Periodontol 1996;67(2):78-85.

8. Lessa FCR, Enoki C, Ito IY, Faria G, Matsumoto MAN, NelsonFilho P. In vivo evaluation of the bacterial contamination and disinfection of acrylic baseplates of removable orthodontic appliances. Am J Orthod Dentofacial Orthop 2007;131(6):705. e11-e17.

9. Vento-Zahra E, De Wever B, Decelis S, Mallia K, Camilleri S. Randomized, double-blind, placebo-controlled trial to test the efficacy of nitradine tablets in maxillary removable orthodontic appliance patients. Quintessence 2011;42(1):37.

10. Gong SQ, Epasinghe J, Rueggeberg FA, Niu LN, Mettenberg D, Yiu CK, et al. An ORMOSIL-containing orthodontic acrylic resin with concomitant improvements in antimicrobial and fracture toughness properties. PloS One 2012;7(8):e42355.

11. Kumar A, Vemula PK, Ajayan PM, John G. Silver-nanoparticle-embedded antimicrobial paints based on vegetable oil. Nat Mater 2008;7(3):236-241. 
12. Pyrek KM. Battling biofilm: surface science, antimicrobials help combat medical device-related infections. Infect Cont Today 2002;42(1):23-25.

13. Simpson K. Using silver to fight microbial attack. Plastics Additives Compounding 2003;5(5):32-35.

14. Ahn S-J, Lee S-J, Kook J-K, Lim B-S. Experimental antimicrobial orthodontic adhesives using nanofillers and silver nanoparticles. Dent Mater 2009;25(2):206-213.

15. Zhao L, Wang H, Huo K, Cui L, Zhang W, Ni H, et al. Antibacterial nano-structured titania coating incorporated with silver nanoparticles. Biomater 2011;32(24):5706-5716.

16. Monteiro DR, Gorup LF, Takamiya AS, de Camargo ER, Barbosa DB. Silver distribution and release from an antimicrobial denture base resin containing silver colloidal nanoparticles. J Prosthodont 2012;21(1):7-15.

17. Acosta-Torres LS, López-Marín LM, Nunez-Anita RE, Hernández-Padrón G, Castaño VM. Biocompatible metaloxide nanoparticles: nanotechnology improvement of conventional prosthetic acrylic resins. J Nanomat 2011;2011:12.

18. Sondi I, Goia DV, Matijević E. Preparation of highly concentrated stable dispersions of uniform silver nanoparticles. J Colloid Interface Sci 2003;260(1):75-81.

19. Riley DK, Classen DC, Stevens LE, Burke JP. A large randomized clinical trial of a silver-impregnated urinary catheter: lack of efficacy and staphylococcal superinfection. Am J Med 1995;98(4):349-356.

20. Crabtree JH, Burchette RJ, Siddiqi RA, Huen IT, Hadnott LL, Fishman A. The efficacy of silver-ion implanted catheters in reducing peritoneal dialysis-related infections. Perit Dial Int 2003;23(4):368-374.

21. Furno F, Morley KS, Wong B, Sharp BL, Arnold PL, Howdle SM, et al. Silver nanoparticles and polymeric medical devices: a new approach to prevention of infection? J Antimicrob Chemother 2004;54(6):1019-1024.

22. Fan C, Chu L, Rawls HR, Norling BK, Cardenas HL, Whang K. Development of an antimicrobial resin-A pilot study. Dent Mater 2011;27(4):322-328.

23. Bjerklin K, Gärskog B, Rönnerman A. Proximal caries increment in connection with orthodontic treatment with removable appliances. J Orthod 1983;10(1):21-24.

24. Gkantidis N, Christou P, Topouzelis N. The orthodonticperiodontic interrelationship in integrated treatment challenges: a systematic review. J Oral Rehabilitation 2010;37(5):377-390.

25. Marsh P. Are dental diseases examples of ecological catastrophes? Microbiol 2003;149(2):279-294.

26. Shah AG, Shetty PC, Ramachandra C, Bhat NS, Laxmikanth S. In vitro assessment of photocatalytic titanium oxide surface modified stainless steel orthodontic brackets for antiadherent and antibacterial properties against Lactobacillus acidophilus. Angle Orthodont 2011;81(6):1028-1035.

27. Seki M, Yamashita Y, Shibata Y, Torigoe H, Tsuda H, Maeno M. Effect of mixed mutans streptococci colonization on caries development. Oral Microbiol Immunol 2006;21(1):47-52.

28. Kassaee M, Akhavan A, Sheikh N, Sodagar A. Antibacterial effects of a new dental acrylic resin containing silver nanoparticles. J Appl Polym Sci 2008;110(3):1699-1703.
29. Bahador A, Lesan S, Kashi N. Effect of xylitol on cariogenic and beneficial oral streptococci: a randomized, double-blind crossover trial. Iran J Microbiol 2012;4(2):75-79.

30. Rompré A, Servais P, Baudart J, de-Roubin M-R, Laurent P. Detection and enumeration of coliforms in drinking water: current methods and emerging approaches. J Microbiol Methods 2002;49(1):31-54.

31. Ashraf R, Shah NP. Selective and differential enumerations of Lactobacillus delbrueckii subsp. bulgaricus, Streptococcus thermophilus, Lactobacillus acidophilus, Lactobacillus casei and Bifidobacterium spp. in yoghurt-A review. Int J Food Microbiol 2011;149(3):194-208.

32. Nelson-Filho P, Valdez RMA, Andrucioli MCD, Saraiva $\mathrm{MCP}$, Feres M, Sorgi CA, et al. Gram-negative periodontal pathogens and bacterial endotoxin in metallic orthodontic brackets with or without an antimicrobial agent: an in vivo study. Am J Orthod Dentofacial Orthop 2011;140(6): e281-e287.

33. Ryu H-S, Bae I-H, Lee K-G, Hwang H-S, Lee K-H, Koh J-T, et al. Antibacterial effect of silver-platinum coating for orthodontic appliances. Angle Orthodont 2011;82(1):151-157.

34. Oei JD, Zhao WW, Chu L, DeSilva MN, Ghimire A, Rawls HR, et al. Antimicrobial acrylic materials with in situ generated silver nanoparticles. J Biomed Mater Res B Appl Biomater 2012;100(2):409-415.

35. Xing M, Shen F, Liu L, Chen Z, Guo N, Wang X, et al. Antimicrobial efficacy of the alkaloid harmaline alone and in combination with chlorhexidine digluconate against clinical isolates of Staphylococcus aureus grown in planktonic and biofilm cultures. Lett Appl Microbiol 2012;54(5):475-482.

36. Saginur R, StDenis M, Ferris W, Aaron SD, Chan F, Lee C, et al. Multiple combination bactericidal testing of staphylococcal biofilms from implant-associated infections. Antimicrob Agents Chemother 2006;50(1):55-61.

37. Lee C-F, Lee C-J, Chen C-T, Huang C-T. $\delta$-Aminolaevulinic acid mediated photodynamic antimicrobial chemotherapy on Pseudomonas aeruginosa planktonic and biofilm cultures. J Photochem Photobiol 2004;75(1):21-25.

38. Surdeau N, Laurent-Maquin D, Bouthors S, Gellé M-P. Sensitivity of bacterial biofilms and planktonic cells to a new antimicrobial agent, Oxsil 320N. J Hosp Infect 2006;62(4): 487-493.

39. Hennig S, Nyunt Wai S, Ziebuhr W. Spontaneous switch to PIA-independent biofilm formation in an ica positive Staphylococcus epidermidis isolate. Int J Med Microbiol 2007;297(2):117-122.

40. O'Gara JP. Ica and beyond: biofilm mechanisms and regulation in Staphylococcus epidermidis and Staphylococcus aureus. FEMS Microbiol lett 2007;270(2):179-188.

41. Brandt O, Mildner M, Egger AE, Groessl M, Rix U, Posch M, et al. Nanoscalic silver possesses broad-spectrum antimicrobial activities and exhibits fewer toxicological side effects than silver sulfadiazine. Nanomed 2012;8(4):478-488.

42. Mei ML, Li Q-l, Chu C-H, Lo EC-M, Samaranayake LP. Antibacterial effects of silver diamine fluoride on multispecies cariogenic biofilm on caries. Ann Clin Microbiol Antimicrob 2013;12(1):4-8. 